\title{
Ufm1 inhibits LPS-induced endothelial cell inflammatory responses through the NF-kB signaling pathway
}

\author{
YUAN-YUAN LI, GUANG-YA ZHANG, JIANG-PING HE, DAN-DAN ZHANG, \\ XIANG-XIN KONG, HUI-MIN YUAN and FENG-LING CHEN \\ Department of Endocrinology, Shanghai Ninth People's Hospital, \\ Shanghai Jiao Tong University School of Medicine, Shanghai 201999, P.R. China
}

Received November 9, 2016; Accepted March 15, 2017

DOI: $10.3892 /$ ijmm.2017.2947

\begin{abstract}
Endothelial cell dysfunction and inflammatory responses are important early contributors to the occurrence and development of atherosclerosis (AS), which still remains to be decoded. Ubiquitin-fold modifier 1 (Ufm1) is a new member of the ubiquitin-like protein family, and its biological function remains largely unknown, particularly in endothelial cell injury and inflammatory responses. In the present study, we showed that Ufm1 was highly expressed in both the nucleus and cytoplasm of human umbilical vein endothelial cells (HUVECs). We also demonstrated that the Ufm1 expression level was increased following lipopolysaccharide (LPS)-induced inflammation in HUVECs. Moreover, overexpression of Ufm1 in HUVECs alleviated the inflammatory responses induced by LPS treatment. Additionally, we found that Ufm1 overexpression inhibited the nuclear translocation of nuclear factor- $\kappa \mathrm{B}(\mathrm{NF}-\kappa \mathrm{B})$ after LPS treatment, suggesting its implication in the LPS/Toll-like receptor 4 (TLR4)/NF- $\mathrm{B}$ pathway. Taken together, in addition to decoding its expression pattern in endothelial cells, we showed for the first time that Ufm1 is upregulated in LPS-induced inflammation and Ufm1 plays an inhibitory role in inflammatory responses by targeting NF- $\kappa \mathrm{B}$ nuclear translocation. Thus, Ufm1 may be a novel gene that protects against inflammatory responses.
\end{abstract}

\section{Introduction}

Atherosclerosis (AS) is a complex process involving numerous cell types and important cell-to-cell interactions that ultimately lead to progression from the 'fatty streak' to formation of more complex atherosclerotic plaques (1-4). The initiation of AS is

Correspondence to: Professor Feng-Ling Chen, Department of Endocrinology, Shanghai Ninth People's Hospital, Shanghai Jiao Tong University School of Medicine, 280 Mo-He Road, Shanghai 201999, P.R. China

E-mail: phdflchen@163.com

Key words: ubiquitin-fold modifier 1, human umbilical vein endothelial cells, lipopolysaccharide, inflammatory responses, nuclear factor-kB multifactorial and caused by a collection of risk factors (3-5). The precise initiating event is unknown; however, dysfunction within the endothelium is thought to be an important early contributor and results in the earliest detectable changes in the life history of an atherosclerotic lesion (1,5-9). The endothelium is crucial for maintenance of vascular homeostasis, ensuring that a balance remains between vasoactive factors controlling its permeability, adhesiveness, and integrity (1,5,7). Endothelial cell (EC) activation or injury by a variety of stimuli including pro-inflammatory cytokines, certain bacterial endotoxins and hemodynamic factors, leads to local thrombosis, loss of vessel barrier function, and rapid and robust leukocyte recruitment $(5,10,11)$. If unchecked, these alterations can contribute to cardiovascular diseases including AS, ischemia/reperfusion injury, rheumatoid arthritis and allograft rejection $(5,6)$. One of the important links between AS and pro-inflammatory endothelial activation is the intrinsic capacity of activated vascular endothelium to synthesize and secrete chemokines, such as tumor necrosis factor- $\alpha$ (TNF- $\alpha$ ), interleukin-1 $\beta$ (IL-1 $\beta$ ), thus generating localized, intercellular autocrine and paracrine signaling loops within the vessel wall $(1,12,13)$. Indeed, cytokines are produced by and act on almost all cells involved in the pathogenesis of AS, participating in all steps of the process, from the early endothelial dysfunction to the late formation and disruption of a vulnerable plaque $(14,15)$. Thus detecting early injury or pro-inflammatory endothelial activation will shed light on mechanisms that are responsible for inflammation/injury-initiated AS.

In recent years, it has been shown that many cellular regulatory processes depend on the post-translational functions of ubiquitin and ubiquitin-like proteins (Ubls), including transcription, DNA repair, signal transduction, autophagy, cell proliferation, differentiation, apoptosis, endoplasmic reticulum (ER) regulation, inflammation, antigen processing and stress responses (16-18). Ubiquitin-fold modifier 1 (Ufm1) has recently been identified as a novel Ubl with a molecular mass of $9.1 \mathrm{kDa}$, and it appears to have a similar tertiary structure to ubiquitin, despite having little (16\%) amino acid sequence identity (19). Similar to the process of protein ubiquitination, Ufm1 is first synthesized in a proform and is cleaved at the C-terminus by the specific cysteine proteases, UfSP1 and UfSP2, to expose the conserved glycine residue that is essential for its subsequent conjugating reac- 
tions $(20,21)$. The mature form of Ufm1 is activated via an E1-activating enzyme, Uba5, and is then conjugated by an E2 enzyme, Ufc1. With the assistance of E3 ligase, Ufm1 is presumed to modify its protein targets $(19,21,22)$. These findings suggest that protein ufmylation is orchestrated through a sophisticated enzymatic pathway, and it represents a novel potential mechanism to regulate protein interaction, localization and function (21).

Ufm1 and its system have been demonstrated to play a significant role in erythroid differentiation (22), cellular growth and development and ER functions (21). It is also suggested that Ufm1 is involved in pathological conditions or diseases, such as tumorigenesis (23-25), ischemic heart diseases (26) and diabetes (27). Despite, to date, the function of Ufm1 remains poorly understood. Previously, both our group (28) and other studies $(27,29)$ indicate that Ufm1 is involved in ER stress, which is a key process of macrophage differentiation and cholesterol deposition in the development of AS (30-32). Our follow-up study also showed that Ufm 1 is markedly upregulated under AS conditions and Ufm1 suppresses foam cell formation via the LXR $\alpha$-dependent pathway (33).

Although the relationship of Ufm1 in ER stress induced-macrophage foam cells, the late stage of AS, has been uncovered, its potential role in initial EC dysfunction or inflammatory responses, the early event in AS, remains unclear. The expression pattern is also unknown. Here, in this study, we evaluated Ufm1 expression in human umbilical vein endothelial cells (HUVECs), as well as the effects of Ufm1 on lipopolysaccharide (LPS)-induced HUVEC inflammation. We found that Ufm1 was expressed in HUVECs and localized in the nucleus and cytoplasm. In LPS-induced HUVEC inflammation, Ufm1 mRNA and protein levels were upregulated. Moreover, Ufm1 overexpression significantly inhibited the expression of LPS-induced inflammatory cytokines by targeting nuclear factor $-\kappa \mathrm{B}(\mathrm{NF}-\kappa \mathrm{B})$ nuclear translocation. Thus, our results demonstrated that Ufm1 plays a significant role in suppressing inflammatory responses via the NF-kB signaling pathway.

\section{Materials and methods}

Cell culture and treatment. HUVECs were obtained from the American Type Culture Collection (ATCC, Rockville, MD, USA) and cultured in Dulbecco's modified Eagle's medium (DMEM) supplemented with 10\% fetal bovine serum (FBS), $100 \mathrm{U} / \mathrm{ml}$ penicillin and $100 \mu \mathrm{g} / \mathrm{ml}$ streptomycin. The cells were incubated in a humidified incubator at $37^{\circ} \mathrm{C}$ with $5 \% \mathrm{CO}_{2}$. When the cells reached $\sim 70-80 \%$ confluency, they were dissociated with trypsinization and subcultured. All of the transient transfections were performed with Lipofectamine 2000 reagent (Invitrogen). For the LPS stimulation, the HUVECs were treated with $100 \mathrm{ng} / \mathrm{ml}$ LPS at a final concentration at the indicated times.

Construction of the Ufm1 plasmid. The Ufm1 cDNA sequence was obtained from 293 cells (obtained from ATCC) by reverse transcription of 293 cell mRNA and PCR amplification as previously described (33). Then the Ufm1 sequence was amplified and cloned into the pcDNA3.1 vector, along with a Flag tag in its N-terminus.
Immunofluorescence. For the immunofluorescence assay, the HUVECs were dissociated into single cells and the dissociated single HUVEC cell suspension was plated onto chamber slides in a 24-well plate. After being cultured for 2 days in complete medium, the HUVECs were then fixed for $30 \mathrm{~min}$ at $4^{\circ} \mathrm{C}$ in $4 \%$ paraformaldehyde (PFA). Then the cells were blocked in $1 \mathrm{X}$ phosphate-buffered saline (PBS) plus $0.3 \%$ Triton $\mathrm{X}-100$ and $10 \%$ normal donkey serum (122346; Jackson ImmunoResearch, West Grove, PA, USA) for $60 \mathrm{~min}$ at room temperature (RT), followed by incubation at $4^{\circ} \mathrm{C}$ overnight in primary antibodies (diluted in blocking buffer). After 3 washes (10 min for each wash) in $1 \mathrm{X}$ PBS plus $0.1 \%$ Triton X-100, the cells were incubated in the fluorescence-conjugated secondary antibodies (1:500 dilution in 1X PBS) plus DAPI for $1 \mathrm{~h}$ at RT. After 3 times washes, the chamber slides were then mounted with mounting medium and imaged. Rabbit anti-Ufm1 antibody (1:1,000; ab109305; Abcam, Cambridge, MA, USA) was used as the primary antibody. Donkey anti-rabbit IgG conjugated with AlexaFluor 594 (1:500; Life Technologies, Grand Island, NY, USA) was used as the secondary antibody. For the negative control, PBS was used to replace the primary antibody and other procedures were identical. Images were captured using a fluorescence microscope.

Real-time quantitative PCR ( $q R T-P C R)$. Total RNA was extracted from cultured cells using TRIzol reagent (Invitrogen), according to the manufacturer's instructions. qRT-PCR was performed using SYBR Premix ExTaq ${ }^{\mathrm{TM}}$ (Takara, Tokyo, Japan) and an Applied Biosystems 7500 Fast real-time PCR system. PCR primer sequences were as follows: Ufm1 forward, 5'-CCT GAAAGTACACCTTTCACAGC-3' and reverse, 5'-CCAGCAG TCTGTGCAGGATT-3'; TNF- $\alpha$ forward, 5'-ATTGCCCTGT GAGGAGGAC-3' and reverse, 5'-TGAGCCAGAAGAGGTTG AGG-3'; IL-6 forward, 5'-CTTCGGTCCAGTTGCCTTCT-3' and reverse, 5'-GTGAGTGGCTGTCTGTGTGG-3'; IL-12 forward, 5'-CTTGTGGCTACCCTGGTCCT-3' and reverse, 5'-GAGTTTGTCTGGCCTTCTGG-3'; IL-1 $\beta$ forward, 5'-GGA TATGGAGCAACAAGTGG-3' and reverse, 5'-ATGTACCAG TTGGGGAACTG-3'; monocyte chemoattractant protein-1 (MCP-1) forward, 5'-CCAATTCTCAAACTGAAGCTCGC-3' and reverse 5'-CTTAGCTGCAGATTCTTGGGTTGTG-3'; and glyceraldehyde 3-phosphate dehydrogenase (GAPDH) forward, 5'-TGCACCACCAACTGCTTAGC-3' and reverse, 5'-GGCAT GGACTGTGGTCATGAG-3'. Individual samples were run in triplicate, and each experiment was repeated at least three times. The $2^{-\Delta \Delta \mathrm{Cq}}$ method was used to analyze the relative changes in gene expression. GAPDH was used as an endogenous normalization control.

Western blot analysis. Western blot analysis was performed as previously described $(28,33)$. Briefly, the HUVECs were dissected, homogenized, and solubilized at $4^{\circ} \mathrm{C}$ in Cell lysis buffer (P0013; Beyotime, Shanghai, China) supplemented with $1 \mathrm{mM}$ PMSF, $50 \mathrm{mM} \mathrm{NaF}, 1 \mathrm{mM} \mathrm{Na} \mathrm{VO}_{4}$ and protease inhibitor. The total protein lysates were separated by sodium dodecyl sulfate-polyacrylamide gel electrophoresis (SDS-PAGE) and analyzed by western blotting. The following antibodies were used: anti-Ufm1 antibody (1:1,000; ab109305; Abcam), anti-nuclear factor- $\kappa \mathrm{B}(\mathrm{NF}-\kappa \mathrm{B})$ p65 (1:1,000; 8242; Cell Signaling Technology, Danvers, MA, USA), anti-GAPDH 


\section{A DAPI/Ufm1/Merged}
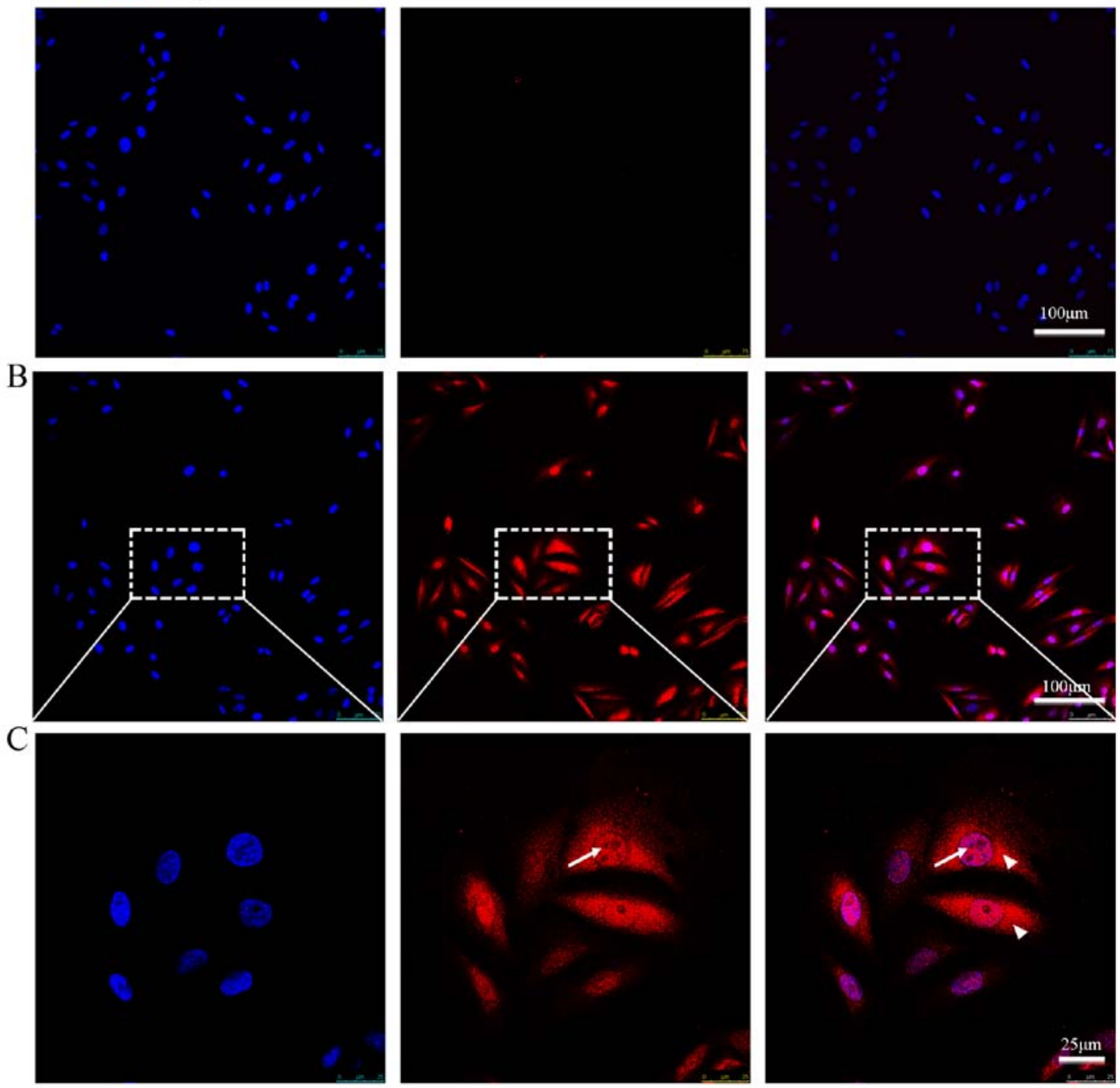

Figure 1. Ubiquitin-fold modifier 1 (Ufm1) is expressed both in the cytoplasm and the nucleus of human umbilical vein endothelial cells (HUVECs). (A-C) The expression of Ufm1 in HUVECs visualized by immunofluorescence (indicated with white arrow). Scale bars: (A and B) $50 \mu \mathrm{m}$ and (C) $25 \mu \mathrm{m}$. The boxes in (B) are shown at a higher magnification in (C). (C) The white arrow indicates the co-localization of Ufm1 with DAPI. The arrow head indicates the expression of Ufm1 in the cytoplasm. Negative control (primary antibody Ufm1 was replaced with PBS) is shown in (A).

(1:5,000; G8795; Sigma-Aldrich, St. Louis, MO, USA), anti$\beta$-actin (1:5,000; MA5-15739; Thermo Fisher Scientific, Waltham, MA, USA) and anti-histone H3 (1:3,000; 4499; Cell Signaling Technology). HRP-conjugated anti-rabbit, antimouse and anti-goat secondary antibodies (A0208, A0216 and A0181) were from Beyotime. Analysis of the data was performed using NIH ImageJ software. The mean density of each band was normalized to the actin or GAPDH signal in the same sample.

For the nuclear and cytoplasmic protein separation experiment (Fig. 5), the protein in the nucleus and cytoplasm were extracted using a nuclear and cytoplasmic protein extraction kit (P0028; Beyotime); then as described above, the nuclear and cytoplasmic protein were analyzed by western blotting with the indicated antibody. $\beta$-actin and histone $\mathrm{H} 3$ were used as loading controls for protein in the cytoplasm and nucleus respectively.

Statistical analysis. All experiments were performed at least three times in triplicate. The results are presented as mean \pm SEM. Statistical differences were determined by the Student's t test for two-group comparisons or ANOVA followed by Tukey's test for multiple comparisons among more than two groups. $\mathrm{P}<0.05$ was considered statistically significant.

\section{Results}

Expression of Ufm1 in HUVECs. To identify whether Ufm1 is expressed in endothelial cells, we performed immunofluorescence on the cultured HUVECs with the Ufm1 antibody. Immunofluorescence assay revealed that endogenous Ufm1 was highly expressed in the HUVECs and was localized both in the cytoplasm and the nucleus (as indicated by its co-localization with DAPI) (Fig. 1).

Ufml is involved in LPS-induced inflammatory responses. Both our previous and other studies have demonstrated that Ufm1 is involved in ER stress (21,26-29); however, whether Ufm1 participates in inflammatory responses remains unknown. In this study, to investigate the association between 


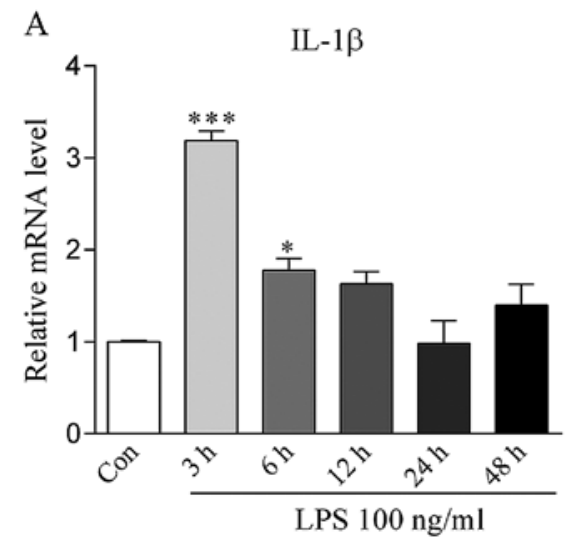

C

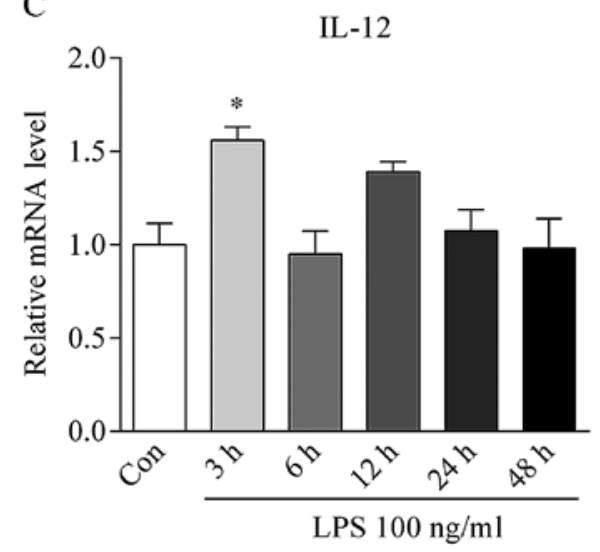

E

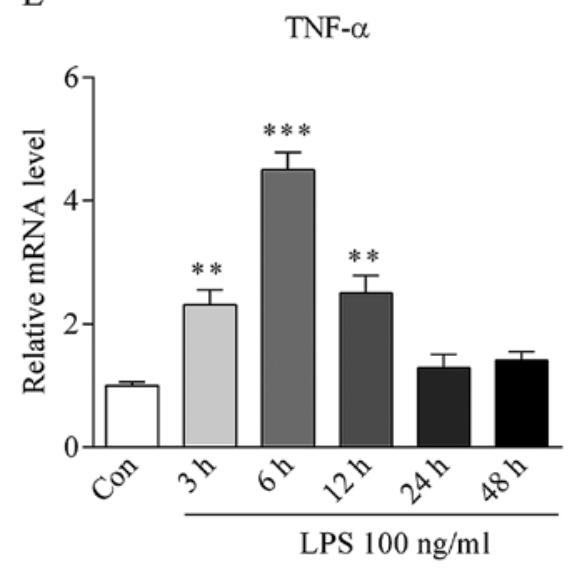

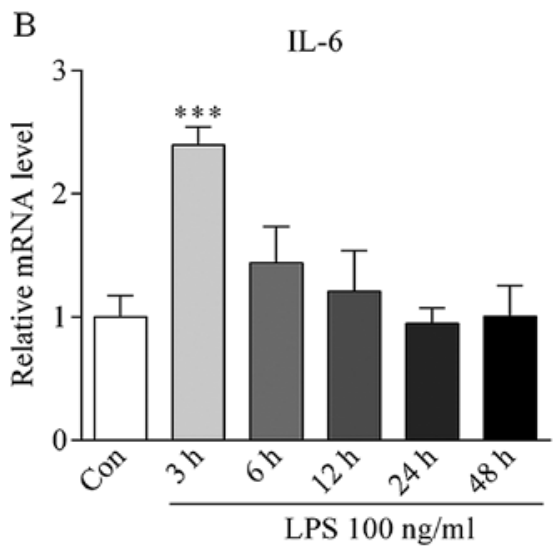

$\mathrm{D}$

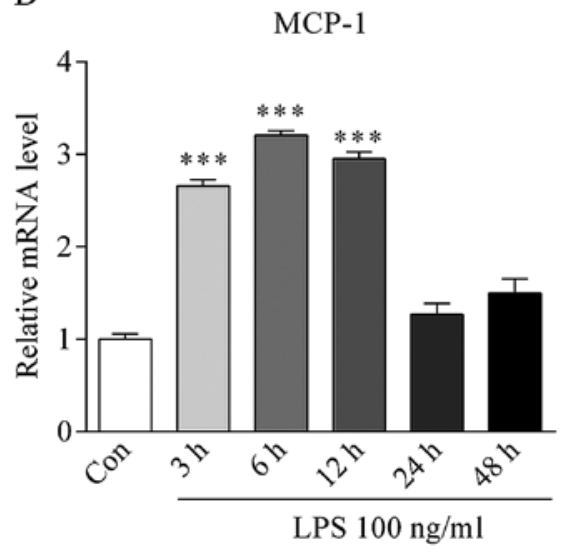

F

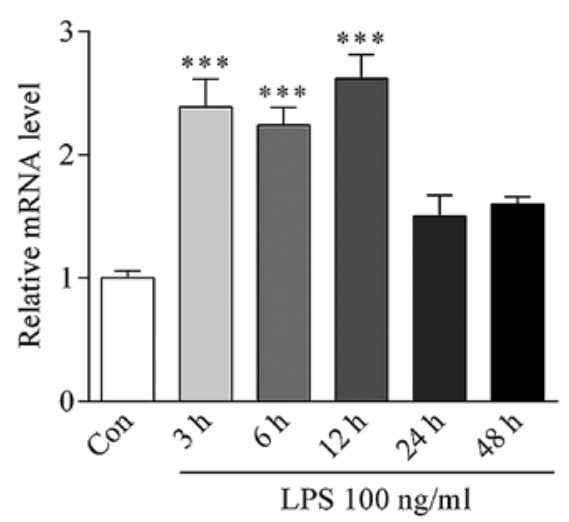

Figure 2. mRNA levels of inflammatory cytokines and ubiquitin-fold modifier 1 (Ufm1) in HUVECs after lipopolysaccharide (LPS) treatment at different

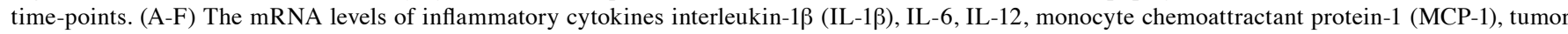
necrosis factor- $\alpha(\mathrm{TNF}-\alpha)$ and Ufm1 were detected by real-time PCR. Glyceraldehyde 3-phosphate dehydrogenase (GAPDH) was used as an internal control. The relative mRNA level was normalized to control group. Data are mean $\pm \operatorname{SEM}(\mathrm{n}=3)$. ${ }^{*} \mathrm{P}<0.05,{ }^{* * *} \mathrm{P}<0.01$ and ${ }^{* * *} \mathrm{P}<0.001$ vs. the control.

Ufm1 expression and endothelial cell injury and inflammatory responses, we examined whether Ufm1 expression is affected in LPS-induced inflammation in HUVECs.

The concentration of $100 \mathrm{ng} / \mathrm{ml}$ LPS was used to induce HUVEC inflammation in vitro. After stimulation for $0,3,6$, 12,24 and $48 \mathrm{~h}$, the mRNA levels of inflammatory cytokines TNF- $\alpha$, IL-6, IL-1 $\beta$, IL-12, MCP-1 were markedly increased and reached a peak at 3-6 h (Fig. 2A-E), suggesting the successful induction of inflammation.

After LPS treatment for $3 \mathrm{~h}$, Ufm1 mRNA expression was increased by $>2.5$-fold in the HUVECs $(\mathrm{P}<0.001$ vs. control) (Fig. 2F). In addition, the Ufm1 protein level was gradually increased from 3 to $24 \mathrm{~h}$ and reached a peak level at 24 h, which was $\sim 3$-fold high compared to the control (Fig. 3).

Together, our data suggest that an upregulation in Ufm1 expression may be associated with LPS-induced HUVEC inflammatory responses.

Overexpression of Ufml inhibits LPS-induced inflammation in HUVECs. To detect the potential role of Ufm1 in inflammatory responses, we first constructed a Ufm1 overexpression plasmid. After transfection into HUVECs, the mRNA level of Ufm1 was significantly increased compared to the control (transfected with the plasmid vector) (Fig. 4A). We also 
A
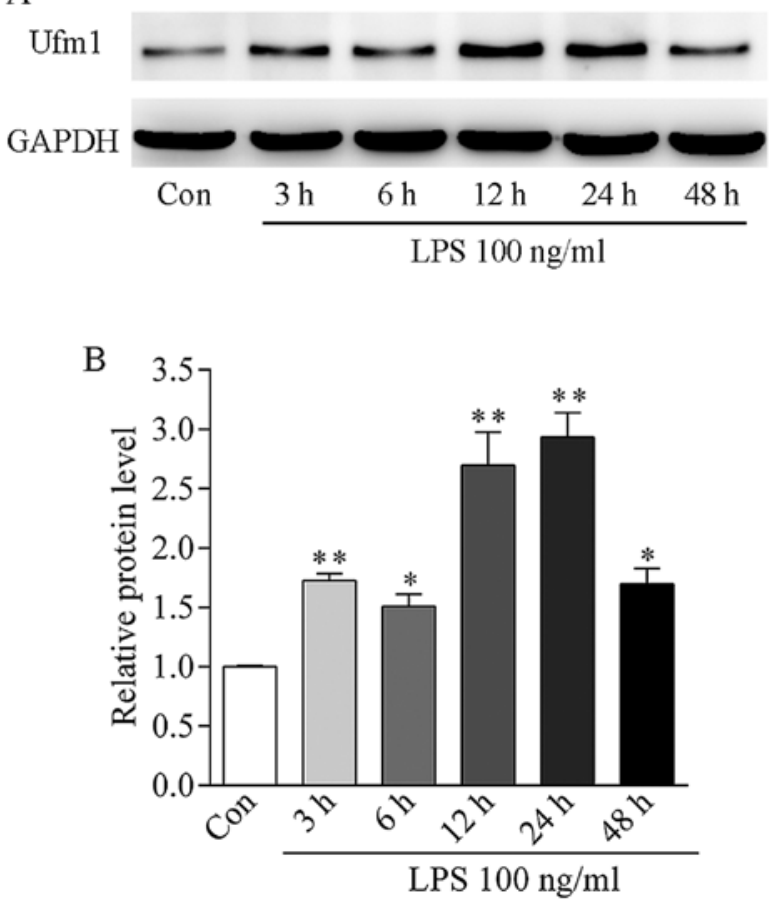

Figure 3. The ubiquitin-fold modifier 1 (Ufm1) protein level is markedly increased in lipopolysaccharide (LPS)-induced inflammatory responses in human umbilical vein endothelial cells (HUVECs). (A) Detection of the protein level of Ufm1 in HUVECs treated with LPS $(100 \mathrm{ng} / \mathrm{ml})$ at different time-points. The same blot was probed with glyceraldehyde 3-phosphate dehydrogenase (GAPDH) antibody as a loading control. (B) Quantification of the protein level in (A) normalized to the control. Data are mean \pm SEM $(n=4) .{ }^{*} \mathrm{P}<0.05$ and ${ }^{* *} \mathrm{P}<0.01$.

confirmed its protein expression by western blotting (WB) using an anti-flag antibody (Fig. 4B).

Since Ufm1 is involved in LPS-induced inflammation (Figs. 2 and 3), it prompted us to further investigate its role in inflammatory responses. After transfection with the Ufm1 overexpression plasmid or vector, the HUVECs were treated with $100 \mathrm{ng} / \mathrm{ml}$ LPS as mentioned above. The mRNA level of cytokines were determined by real-time PCR. In comparison to the control, LPS significantly increased the inflammatory cytokine expression (Fig. 4C). After Ufm1 overexpression, the expression of the inflammatory cytokines was markedly reduced, especially TNF- $\alpha$, IL- $1 \beta$ and IL-12 (Fig. 4C).

Our data indicated that overexpression of Ufm1 can inhibit LPS-induced inflammatory responses in HUVECs.

Ufml suppresses inflammatory responses via the LPS-induced $N F-\kappa B$ pathway in HUVECs. As Ufm1 is involved in inflammation and Ufm 1 overexpression can inhibit LPS-induced inflammatory responses in HUVECs (Figs. 2 and 4), it raised the question of how Ufm1 exerts its function. Given the fact that LPS-induced inflammation is through Toll-like receptor (TLR) signaling, and that $\mathrm{NF}-\kappa \mathrm{B}$ is activated and enters into the nucleus to regulate the induced transcription of pro-inflammatory genes (34), a process known as NF- $\mathrm{B}$ nuclear translocation, we analyzed the effect of Ufm1 on the cellular distribution of $\mathrm{NF}-\kappa \mathrm{B}$. The cytoplasmic and nuclear proteins were separated as indicated by $\beta$-actin and
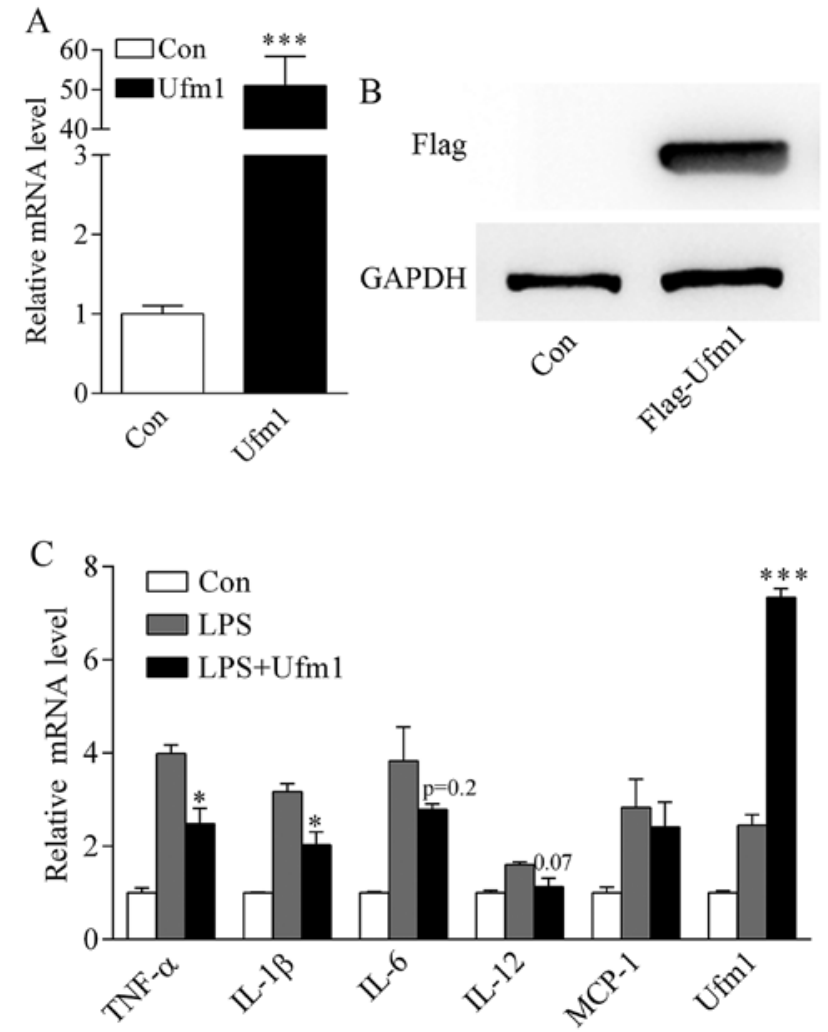

Figure 4. Overexpression of ubiquitin-fold modifier 1 (Ufm1) inhibits lipopolysaccharide (LPS)-induced inflammatory responses in human umbilical vein endothelial cells (HUVECs). (A and B) Verification of Ufm 1 overexpression after transfection with Flag-Ufm1 by real-time PCR and western blotting (WB). Data are mean \pm SEM $(n=4)$. ${ }^{* * *} \mathrm{P}<0.001$ vs. the control. (C) The relative mRNA levels of tumor necrosis factor- $\alpha$ (TNF- $\alpha$ ), interleukin-6 (IL-6), IL-1 $\beta$, IL-12, monocyte chemoattractant protein-1 (MCP-1) and Ufm1 were detected by real-time PCR in the control, LPS and LPS+Ufm1 groups. Data are mean $\pm \operatorname{SEM}(n=3) .{ }^{*} \mathrm{P}<0.05$ and ${ }^{* * *} \mathrm{P}<0.001$ vs. the LPS group.

histone H3. Upon LPS treatment, the protein level of NF- $\kappa \mathrm{B}$ in the nucleus was significantly increased while it was decreased in the cytoplasm (Fig. 5A and B). Interestingly, we found that Ufm1 overexpression reversed the role of LPS, resulting in decreased expression of $\mathrm{NF}-\kappa \mathrm{B}$ in the nucleus (Fig. 5A and $\mathrm{B}$ ). Consistently, immunofluorescence staining of p65 also indicated that upon LPS treatment, the NF- $\kappa \mathrm{B}$ protein translocated to the nucleus (Fig. 5C). However, Ufm1 overexpression abolished the phenotype induced by LPS, resulting in decreased expression of NF- $\mathrm{NB}$ in the nucleus (Fig. 5C). Based on these findings, we propose that Ufm1 can decrease the nuclear translocation of NF- $\mathrm{BB}$, thus further inhibiting the expression of target inflammatory cytokines.

\section{Discussion}

In the present study, we investigated the expression pattern and potential biological function of Ufm 1 in ECs. We found that Ufm1 was expressed in both the nucleus and the cytoplasm of HUVECs. Along with the increase in inflammatory cytokines, Ufm1 expression was markedly upregulated in response to the inflammatory response induced by LPS in the HUVECs. Further investigation revealed that overexpression of Ufm1 attenuated the LPS-induced inflammatory response. More importantly, we found that Ufm1 inhibited the expression of 
A

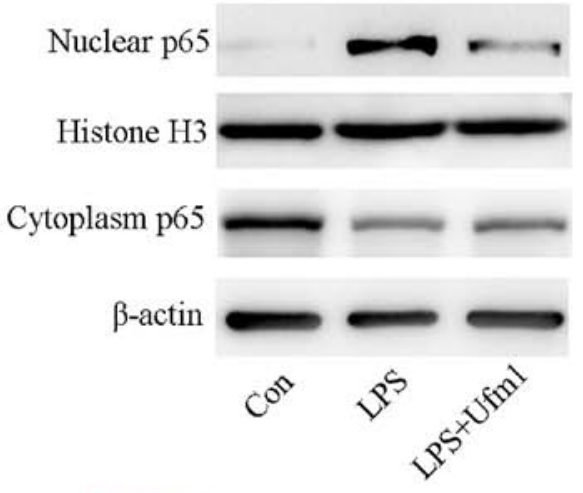

C p65/DAPI/Merged
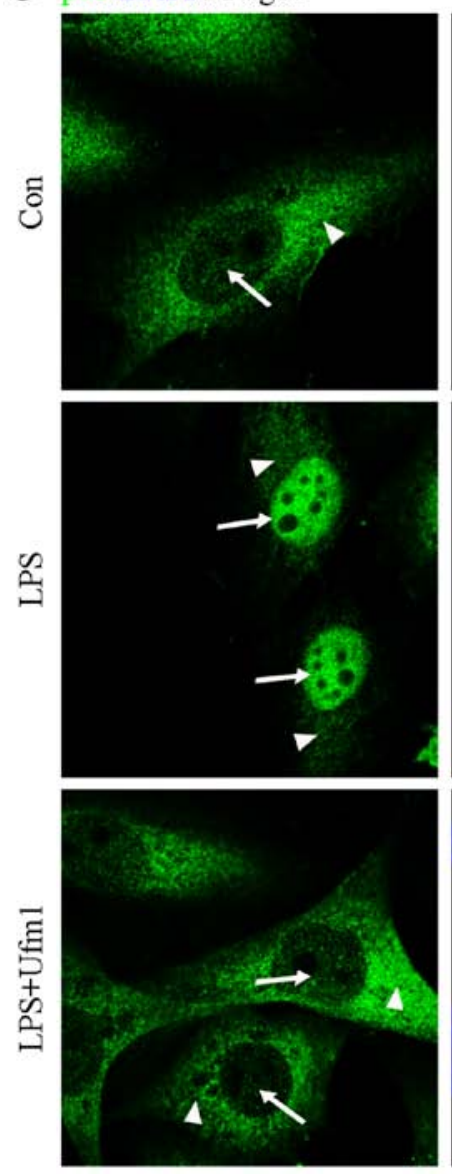
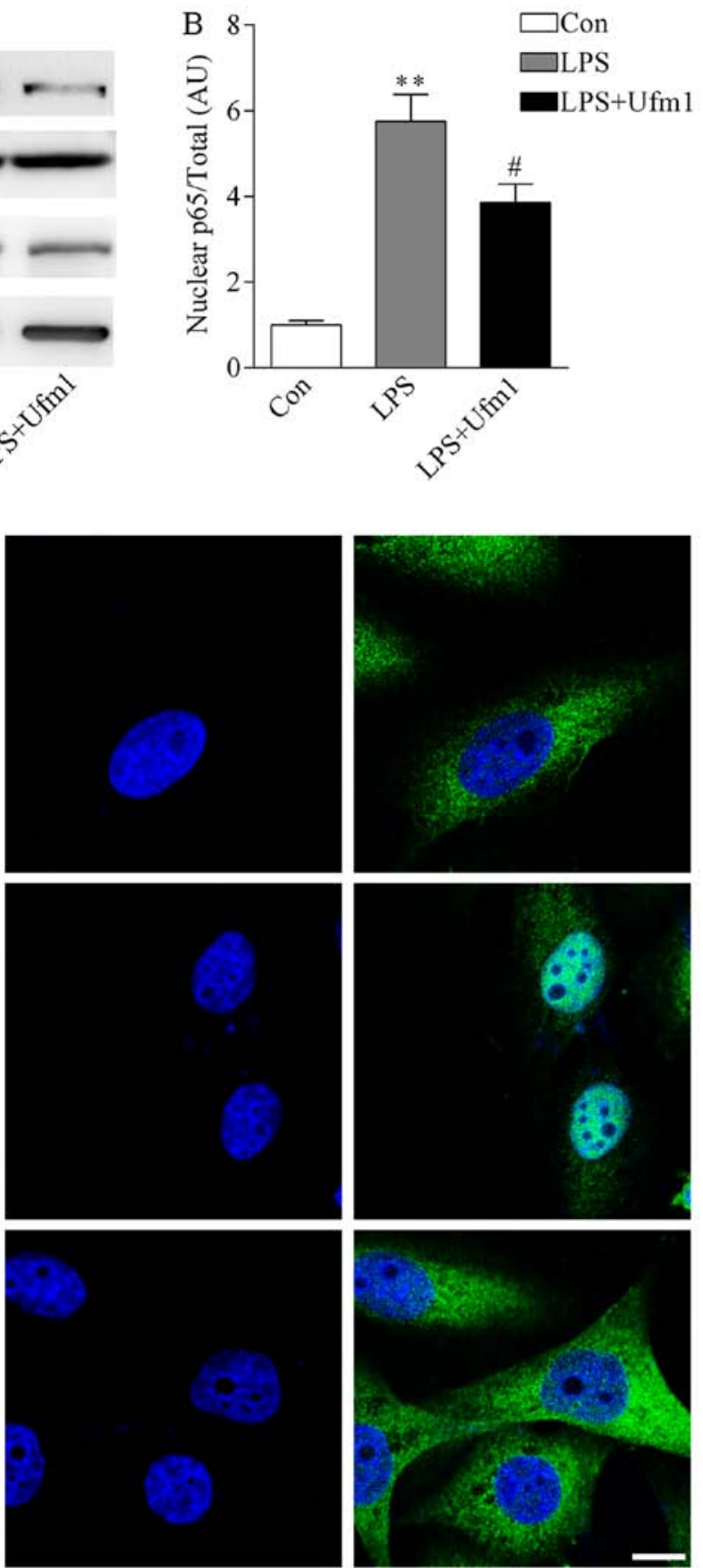

Figure 5. Ubiquitin-fold modifier 1 (Ufm1) overexpression inhibits lipopolysaccharide (LPS)-induced nuclear factor- $\kappa \mathrm{B}$ (NF- $\kappa \mathrm{B})$ nuclear translocation. (A and B) The expression and quantification of p65 protein in the cytoplasm and nucleus in the control, LPS, and LPS+Ufm1 groups. $\beta$-actin and histone H3 were used as loading controls for protein in the cytoplasm and nucleus, respectively. Data are mean $\pm \mathrm{SEM}\left(\mathrm{n}=3\right.$ ). ${ }^{* *} \mathrm{P}<0.01 \mathrm{vs}$. control; ${ }^{\#} \mathrm{P}<0.05 \mathrm{vs}$. LPS group. (C) Immunofluorescence to detect the expression of p65 protein in the cytoplasm and nucleus in the control, LPS, and LPS+Ufm1 groups. The white arrowhead and white arrow indicate the expression of p65 in the cytoplasm and nucleus, respectively. Scale bar, $10 \mu \mathrm{m}$.

inflammatory cytokines through the LPS-induced NF- $\mathrm{BB}$ pathway (Fig. 6).

Ufm1 is a new member of the Ubl family, whose biological functions are poorly understood, particularly in ECs. It has been reported that Ufm1 is expressed in many tissues and cell lines $(19,27)$ and that upregulation of Ufm1 expression is linked with the activation of the ER stress response in AS and diabetes $(27,28,33,35)$. Our previous studies also demonstrated that Ufm1 is involved in AS conditions (28,33). In addition, we found that Ufm1 protects against ER stress-induced apoptosis in macrophages as well as oxidized low-density lipoprotein
(oxLDL)-induced foam cells $(28,33)$, both of which are key regulators in macrophage differentiation and cholesterol deposition in the development of AS (30-32). Here, we investigate Ufm1 expression and further uncovered a novel role of Ufm1 particularly in EC inflammation, which provides new insight into the early stage of AS.

LPS, a unique glycolipid contained in the outer wall of Gram-negative bacteria, can cause endothelial dysfunction and inflammation, which have been demonstrated to be associated with AS (36-38). In this study, we used $100 \mathrm{ng} / \mathrm{ml}$ LPS to induce inflammation and endothelial dysfunction. After 


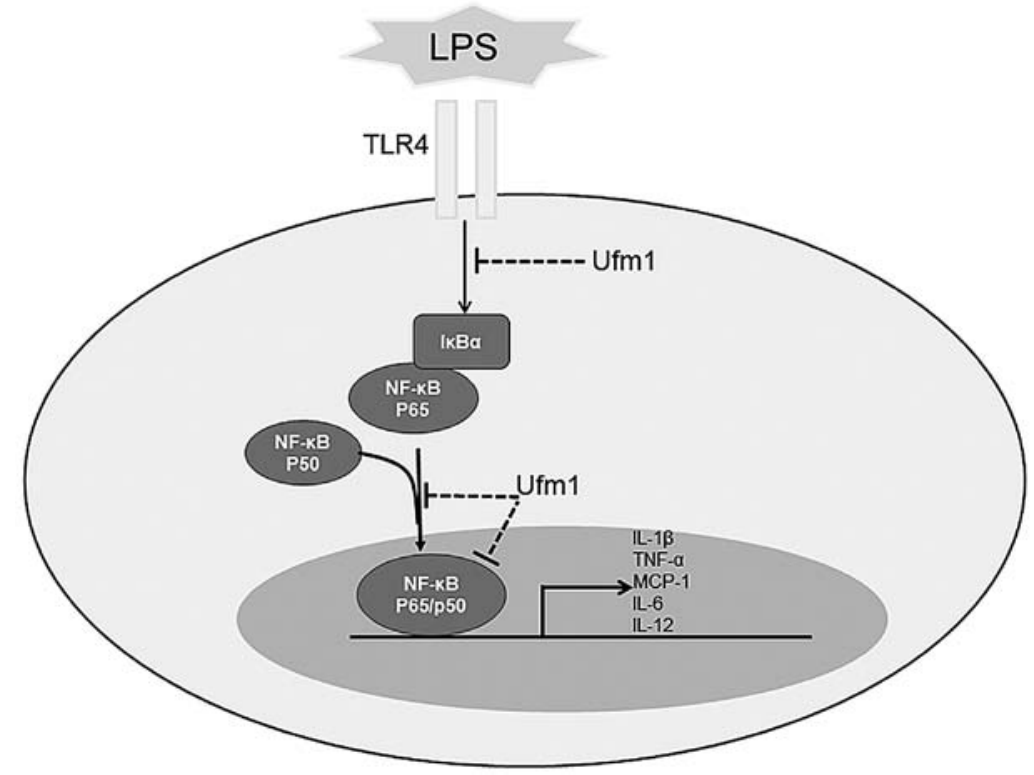

Figure 6. Schematic drawing demonstrating the previously uncharacterized mechanism involved in the inhibition of lipopolysaccharide (LPS)-induced inflammatory responses by ubiquitin-fold modifier 1 (Ufm1) through the nuclear factor- $\mathrm{kB}(\mathrm{NF}-\mathrm{\kappa B})$ pathway.

LPS treatment, Ufm1 expression was upregulated, together with an upregulation of the expression of inflammatory genes including TNF- $\alpha$, IL-6, IL-1 $\beta$, IL-12 and MCP-1 (Fig. 2), suggesting that Ufm1 is involved in EC inflammation. The LPS-TLR4/NF- $\kappa \mathrm{B}$ signaling pathway is a major player in the regulation of diverse biological processes, including immune responses, cell proliferation and inflammation (34,39-41). $\mathrm{NF}-\kappa \mathrm{B}$ is a complex of dimeric subunits that belong to the

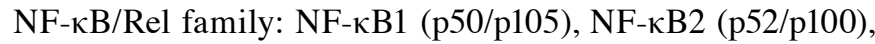
p65 (RelA), RelB and c-Rel $(34,39)$. Normally, $N F-\kappa B$ is inactive and resides in the cytoplasm, where it is sequestered by inhibitors of $\kappa \mathrm{B}(\mathrm{I} \kappa \mathrm{B})$, of which the most important are $\mathrm{I} \kappa \mathrm{B} \alpha, \mathrm{I} \kappa \mathrm{B} \beta$ and $\mathrm{I} \kappa \mathrm{B} \varepsilon(34,39,40)$. When cells are stimulated by cytokines or LPS, they bind to TLR4 assisted by other proteins. Then the TLR4 protein complex initiates recruitment of other proteins $(42,43)$, leading to the phosphorylation of I $\kappa$ Bs at specific serine residues by I $\kappa$ B kinase $(39,44)$. Upon phosphorylation and ubiquitin-dependent degradation of $\mathrm{I} \kappa \mathrm{B} \alpha, \mathrm{NF}-\kappa \mathrm{B}$ translocates to the nucleus and functions as a transcription factor (39). These events lead to the activation of the NF- $\kappa \mathrm{B}$ cascade for the inflammatory response.

In this study, we found that after LPS treatment, $\mathrm{NF}-\kappa \mathrm{B}$ was activated and translocated to the nucleus (Fig. 5), together with upregulation of expression of its target inflammatory genes including TNF- $\alpha$, IL-6, IL-1 $\beta$, IL-12 and MCP-1 (Fig. 2). Moreover, overexpression of Ufm1 prevented the $\mathrm{NF}-\kappa \mathrm{B}$ nuclear translocation indicated by the decreased ratio of $\mathrm{p} 65$ in the nucleus to total (Fig. 5), resulting in decreased proinflammatory cytokine expression (Fig. 4). Together, our data indicated that Ufm1 can inhibit pro-inflammatory responses through the LPS-TLR4/NF- $\mathrm{B}$ pathway in HUVECs. In addition, we also found that in response to LPS treatment, Ufm1 expression was upregulated (Figs. 2F and 3), suggesting a compensatory response in EC inflammation. This is consistent with our previous study that Ufm1 is also increased in ER stress and its overexpression plays a protective role in suppressing foam cell formation and ER stress $(28,33)$.
Recently, Ufm1 modification (ufmylation) has emerged as a novel post-translational modification that plays essential roles in several physiological and pathological processes (21). However, there are still limitations to our study. Because of the difficulty in identifying substrate proteins, the study of the Ufm 1 cascade is still in its infancy and its functions are yet to be completely understood. Regarding the fact that upon phosphorylation and ubiquitin-dependent degradation of $\mathrm{I} \kappa \mathrm{B} \alpha, \mathrm{NF}-\kappa \mathrm{B}$ translocates to the nucleus and functions as a transcription factor $(34,39)$, it is possible that $\mathrm{I} \kappa \mathrm{B} \alpha$ may be a potential substrate of Ufm1 and Ufm1 inhibits the NF- $\kappa \mathrm{B}$ nuclear translocation through I $\kappa \mathrm{B} \alpha$ ufmylation. Or possibly $\mathrm{NF}-\kappa \mathrm{B}$ is the target of Ufm1 and ufmylated NF- $\kappa \mathrm{B}$ can inhibit its role in promoting the transcription of target gene expression. Still we cannot exclude other possibilities, since there are other substrates of Ufm1 which may serve as a bridge in mediating Ufm1 to NF- $\kappa \mathrm{B}$ translocation. For example, in a recent study, Yoo et al found that Ufm1 may also ufmylate LZAP (24), which is a putative tumor suppressor and inhibits the $\mathrm{NF}-\kappa \mathrm{B}$ pathway $(21,45,46)$. Thus, identification of the precise ufmylated substrates involved in LPS-induced EC inflammation will help us to better understand the role of Ufm1 in the initiation and progression of AS. How Ufm1 exerts its role in the NF- $\kappa$ B pathway, identification of ufmylated substrates and whether subsequent ufmylation participates in the protective action of Ufm1 against pro-inflammatory response in ECs require further investigation.

In conclusion, this study found that Ufm1 is expressed in HUVECs and is localized in the nucleus and cytoplasm. Furthermore, our study provides new insight into the anti-inflammatory properties of Ufm1, which reduces proinflammatory cytokine expression by regulating the NF-kB signaling pathway. On the basis of our in vitro findings, we anticipate that Ufm1 may serve as a potential molecular target for developing novel therapeutic strategies that protect ECs from dysfunction, suppress inflammation and subsequently suppress the initial occurrence and development of AS. 
Moreover, in vivo studies exploring the functions of Ufm1 in AS are still needed to provide further evidence for the importance of Ufm1 in the pathogenesis of AS.

\section{Acknowledgements}

This study was supported by the National Natural Science Foundation of China (no. 81270908).

\section{References}

1. Gimbrone MA Jr and García-Cardeña G: Endothelial cell dysfunction and the pathobiology of atherosclerosis. Circ Res 118: 620-636, 2016.

2. Libby P, Ridker PM and Hansson GK; Leducq Transatlantic Network on Atherothrombosis: Inflammation in atherosclerosis: From pathophysiology to practice. J Am Coll Cardiol 54: 2129-2138, 2009.

3. Hansson GK: Inflammation, atherosclerosis, and coronary artery disease. N Engl J Med 352: 1685-1695, 2005.

4. Ross R: Atherosclerosis is an inflammatory disease. Am Heart J 138: S419-S420, 1999

5. Otsuka F, Finn AV, Yazdani SK, Nakano M, Kolodgie FD and Virmani R: The importance of the endothelium in atherothrombosis and coronary stenting. Nat Rev Cardiol 9: 439-453, 2012.

6. Pober JS and Sessa WC: Evolving functions of endothelial cells in inflammation. Nat Rev Immunol 7: 803-815, 2007.

7. Rao RM, Yang L, Garcia-Cardena G and Luscinskas FW: Endothelial-dependent mechanisms of leukocyte recruitment to the vascular wall. Circ Res 101: 234-247, 2007.

8. Stary HC: Natural history and histological classification of atherosclerotic lesions: An update. Arterioscler Thromb Vasc Biol 20: 1177-1178, 2000.

9. Virmani R, Kolodgie FD, Burke AP, Farb A and Schwartz SM: Lessons from sudden coronary death: A comprehensive morphological classification scheme for atherosclerotic lesions. Arterioscler Thromb Vasc Biol 20: 1262-1275, 2000.

10. Gimbrone MA Jr: Endothelial dysfunction, hemodynamic forces, and atherosclerosis. Thromb Haemost 82: 722-726, 1999.

11. Zhang C: The role of inflammatory cytokines in endothelial dysfunction. Basic Res Cardiol 103: 398-406, 2008.

12. Pober JS, Bevilacqua MP, Mendrick DL, Lapierre LA, Fiers W and Gimbrone MA Jr: Two distinct monokines, interleukin 1 and tumor necrosis factor, each independently induce biosynthesis and transient expression of the same antigen on the surface of cultured human vascular endothelial cells. J Immunol 136: 1680-1687, 1986.

13. Libby P, Ordovas JM, Auger KR, Robbins AH, Birinyi LK and Dinarello CA: Endotoxin and tumor necrosis factor induce interleukin-1 gene expression in adult human vascular endothelial cells. Am J Pathol 124: 179-185, 1986.

14. Tedgui A and Mallat Z: Cytokines in atherosclerosis: Pathogenic and regulatory pathways. Physiol Rev 86: 515-581, 2006.

15. Tousoulis D, Oikonomou E, Economou EK, Crea F and Kaski JC: Inflammatory cytokines in atherosclerosis: Current therapeutic approaches. Eur Heart J 37: 1723-1732, 2016.

16. Hochstrasser M: Origin and function of ubiquitin-like proteins. Nature 458: 422-429, 2009.

17. Kerscher O, Felberbaum R and Hochstrasser M: Modification of proteins by ubiquitin and ubiquitin-like proteins. Annu Rev Cell Dev Biol 22: 159-180, 2006.

18. Weissman AM: Themes and variations on ubiquitylation. Nat Rev Mol Cell Biol 2: 169-178, 2001.

19. Komatsu M, Chiba T, Tatsumi K, Iemura S, Tanida I, Okazaki N, Ueno T, Kominami E, Natsume T and Tanaka K: A novel proteinconjugating system for Ufm1, a ubiquitin-fold modifier. EMBO J 23: 1977-1986, 2004.

20. Kang SH, Kim GR, Seong M, Baek SH, Seol JH, Bang OS, Ovaa $\mathrm{H}$, Tatsumi $\mathrm{K}$, Komatsu M, Tanaka $\mathrm{K}$, et al: Two nove ubiquitin-fold modifier 1 (Ufm1)-specific proteases, UfSP1 and UfSP2. J Biol Chem 282: 5256-5262, 2007.

21. Daniel J and Liebau E: The ufm1 cascade. Cells 3: 627-638, 2014

22. Tatsumi K, Sou YS, Tada N, Nakamura E, Iemura S, Natsume T, Kang SH, Chung $\mathrm{CH}$, Kasahara M, Kominami E, et al: A novel type of E3 ligase for the Ufm1 conjugation system. J Biol Chem 285: 5417-5427, 2010.
23. Shiwaku H, Yoshimura N, Tamura T, Sone M, Ogishima S, Watase K, Tagawa K and Okazawa H: Suppression of the novel ER protein Maxer by mutant ataxin-1 in Bergman glia contributes to non-cell-autonomous toxicity. EMBO J 29: 2446-2460, 2010.

24. Yoo HM, Kang SH, Kim JY, Lee JE, Seong MW, Lee SW, Ka SH, Sou YS, Komatsu M, Tanaka K, et al: Modification of ASC1 by UFM1 is crucial for ER $\alpha$ transactivation and breast cancer development. Mol Cell 56: 261-274, 2014.

25. Kim CH, Nam HS, Lee EH, Han SH, Cho HJ, Chung HJ, Lee NS, Choi SJ, Kim H, Ryu JS, et al: Overexpression of a novel regulator of p120 catenin, NLBP, promotes lung adenocarcinoma proliferation. Cell Cycle 12: 2443-2453, 2013.

26. Azfer A, Niu J, Rogers LM, Adamski FM and Kolattukudy PE: Activation of endoplasmic reticulum stress response during the development of ischemic heart disease. Am J Physiol Heart Circ Physiol 291: H1411-H1420, 2006.

27. Lemaire K, Moura RF, Granvik M, Igoillo-Esteve M, Hohmeier HE, Hendrickx N, Newgard CB, Waelkens E, Cnop M and Schuit F: Ubiquitin fold modifier 1 (UFM1) and its target UFBP1 protect pancreatic beta cells from ER stress-induced apoptosis. PLoS One 6: e18517, 2011.

28. Hu X, Pang Q, Shen Q, Liu H, He J, Wang J, Xiong J, Zhang H and Chen F: Ubiquitin-fold modifier 1 inhibits apoptosis by suppressing the endoplasmic reticulum stress response in Raw264.7 cells. Int J Mol Med 33: 1539-1546, 2014.

29. Zhang Y, Zhang M, Wu J, Lei G and Li H: Transcriptional regulation of the Ufm1 conjugation system in response to disturbance of the endoplasmic reticulum homeostasis and inhibition of vesicle trafficking. PLoS One 7: e48587, 2012.

30. Oh J, Riek AE, Weng S, Petty M, Kim D, Colonna M, Cella M and Bernal-Mizrachi C: Endoplasmic reticulum stress controls M2 macrophage differentiation and foam cell formation. J Biol Chem 287: 11629-11641, 2012.

31. Tabas I: The role of endoplasmic reticulum stress in the progression of atherosclerosis. Circ Res 107: 839-850, 2010.

32. Tabas I: Macrophage apoptosis in atherosclerosis: Consequences on plaque progression and the role of endoplasmic reticulum stress. Antioxid Redox Signal 11: 2333-2339, 2009.

33. Pang Q, Xiong J, Hu XL, He JP, Liu HF, Zhang GY, Li YY and Chen FL: UFM1 Protects macrophages from oxLDL-induced foam cell formation through a liver $\mathrm{X}$ receptor $\alpha$ dependent pathway. J Atheroscler Thromb 22: 1124-1140, 2015.

34. Tak PP and Firestein GS: NF-kappaB: A key role in inflammatory diseases. J Clin Invest 107: 7-11, 2001.

35. Lu H, Yang Y, Allister EM, Wijesekara N and Wheeler MB The identification of potential factors associated with the development of type 2 diabetes: A quantitative proteomics approach. Mol Cell Proteomics 7: 1434-1451, 2008.

36. Huang B, Chen $\mathrm{H}$ and Fan M: Inhibition of TLR4 signaling pathway: Molecular treatment strategy of periodontitis-associated atherosclerosis. Med Hypotheses 70: 614-617, 2008.

37. Lin MI and Sessa WC: Vascular endothelial growth factor signaling to endothelial nitric oxide synthase: More than a FLeeTing moment. Circ Res 99: 666-668, 2006.

38. Stoll LL, Denning GM and Weintraub NL: Potential role of endotoxin as a proinflammatory mediator of atherosclerosis. Arterioscler Thromb Vasc Biol 24: 2227-2236, 2004.

39. Baldwin AS Jr: The NF-kappa B and I kappa B proteins: New discoveries and insights. Annu Rev Immunol 14: 649-683, 1996.

40. Sen R and Baltimore D: Inducibility of kappa immunoglobulin enhancer-binding protein Nf-kappa B by a posttranslational mechanism. Cell 47: 921-928, 1986.

41. Barnes PJ and Karin M: Nuclear factor-kappaB: A pivotal transcription factor in chronic inflammatory diseases. N Engl J Med 336: 1066-1071, 1997.

42. Fitzgerald KA and Chen ZJ: Sorting out Toll signals. Cell 125: 834-836, 2006.

43. Li H and Sun B: Toll-like receptor 4 in atherosclerosis. J Cell Mol Med 11: 88-95, 2007.

44. Rakonczay Z Jr, Hegyi P, Takács T, McCarroll J and Saluja AK: The role of NF-kappaB activation in the pathogenesis of acute pancreatitis. Gut 57: 259-267, 2008.

45. Gusarova GA, Wang IC, Major ML, Kalinichenko VV, Ackerson T, Petrovic V and Costa RH: A cell-penetrating ARF peptide inhibitor of FoxM1 in mouse hepatocellular carcinoma treatment. J Clin Invest 117: 99-111, 2007.

46. Xi P, Ding D, Zhou J, Wang M and Cong YS: DDRGK1 regulates $\mathrm{NF}-\kappa \mathrm{B}$ activity by modulating $\mathrm{I} \kappa \mathrm{B} \alpha$ stability. PLoS One 8 : e64231, 2013. 